Matrices with Identical Sets of Neighbors Author(s): Imre Bárány and Herbert Scarf

Source: Mathematics of Operations Research, Vol. 23, No. 4 (Nov., 1998), pp. 863-873 Published by: INFORMS

Stable URL: http://www.jstor.org/stable/3690635

Accessed: 19/10/2013 05:27

Your use of the JSTOR archive indicates your acceptance of the Terms \& Conditions of Use, available at http://www.jstor.org/page/info/about/policies/terms.jsp

JSTOR is a not-for-profit service that helps scholars, researchers, and students discover, use, and build upon a wide range of content in a trusted digital archive. We use information technology and tools to increase productivity and facilitate new forms of scholarship. For more information about JSTOR, please contact support@jstor.org. 


\title{
MATRICES WITH IDENTICAL SETS OF NEIGHBORS
}

\author{
IMRE BÁRÁNY AND HERBERT SCARF
}

Given a generic $m$ by $n$ matrix $A$, a lattice point $h$ in $\mathbb{Z}^{n}$ is a neighbor of the origin if the body $\{x: A x \leq b\}$, with $b_{i}=\max \left\{0, a_{i} h\right\}, i=1, \ldots, m$, contains no lattice point other than 0 and $h$. The set of neighbors, $N(A)$, is finite and 0 -symmetric. We show that if $A^{\prime}$ is another matrix of the same size with the property that $\operatorname{sign} a_{i} h=\operatorname{sign} a_{i}^{\prime} h$ for every $i$ and every $h \in N(A)$, then $A^{\prime}$ has precisely the same set of neighbors as $A$. The collection of such matrices is a polyhedral cone, described by a finite set of linear inequalities, each such inequality corresponding to a generator of one of the cones $C_{i}=\operatorname{pos}\left\{h \in N(A): a_{i} h<0\right\}$. Computational experience shows that $C_{i}$ has "few" generators. We demonstrate this in the first nontrivial case $n=3, m=4$.

1. Introduction. Test sets for integer programming were introduced by Graver (1975) and Scarf (1986). They provide a way of telling if a feasible solution $z \in \mathbb{Z}^{n}$ is optimal or not by checking, for each $h$ in the test set, whether $z+h$ is feasible and yields an improved value of the objective function.

The test set of Scarf, the set of neighbors of the origin, is associated with a matrix $A$ of size $m$ by $n$, and is applied to the class of problems of the form

$$
\min a_{1} z
$$

$$
\text { subject to } a_{i} z \leq b_{i}(i=2, \ldots, m), \quad z \in \mathbb{Z}^{n}
$$

in which a single row of $A$ becomes the objective, and the remaining rows are used, with arbitrary $b_{i}$, to form the constraints.

For each lattice point $h \in \mathbb{Z}^{n}$, the smallest body of the form

$$
K_{b}=\left\{x \in R^{n}: A x \leq b\right\}
$$

containing 0 and $h$ is given by $b_{i}=\max \left\{0, a_{i} h\right\}$, for $i=1,2, \ldots, m$. We designate this body by $\langle 0, h\rangle$. The lattice point $h \in \mathbb{Z}^{n}(h \neq 0)$ is defined to be a neighbor of the origin if $\langle 0, h\rangle$ contains no lattice points in its interior. The collection of such neighbors is denoted by $N(A)$. Note that in this definition the special role of $a_{i}$ as the objective function has disappeared.

In the next section we introduce various conditions on $A$ to ensure that $N(A)$ is a test set for the integer programs $(1.1)$, or that $N(A)$ is nonempty and finite. Finiteness of $N(A)$ is proved in quantitative form (Theorem 3 ). Our main result (Theorem 1) characterizes matrices with identical sets of neighbors (and identical sign-pattern of $A h$ for each neighbour). It turns out that this collection of matrices $C(A)$ is a polyhedral set determined by the cones

$$
C_{i}=\operatorname{pos}\left\{h \in N(A): a_{i} h<0\right\}
$$

Received August 13, 1996; revised: March 31, 1998.

AMS 1991 subject classification. Primary: 90C10.

OR/MS subject classification. Primary: Programming/Integer.

Key words. Test sets for integer programming, polyhedral sets of matrices.

863 
where $A$ is a generic (cf. §2) matrix. $C(A)$ has a product structure since the rows of the matrices in it vary in the interior of $C_{i}^{*}$, the polar of $C_{i}$, independently of each other.

Computational experience and some theoretical results (cf. Remark in \$2) indicate that $C_{i}$ has "few" generators. We demonstrate this (Theorem 2) in the first nontrivial case $n$ $=3, m=4$. We also show that the generators form the Hilbert basis of the cone $C_{i}$. The proof is based on properties of the neighbors and of 3-dimensional lattices.

2. Results. We assume throughout that the rank of $A$ is $n$. Notice first that $N(A)$ is symmetric about the origin. This follows from $\langle 0, h\rangle-h=\langle 0,-h\rangle$.

Next, we need to formulate various conditions on the matrix $A$. A convenient way to do so is to consider the dual feasible region

$$
D(A)=\left\{y \in R^{m}: y A=0, y \geq 0\right\} .
$$

The first condition we need is

(A1) There is $y \in D(A)$ with $y_{i}>0(\forall i)$.

This is equivalent to saying that $K_{b}$ is bounded for every $b$, or that $0 \in \operatorname{int} \operatorname{conv}\left\{a_{1}, \ldots\right.$, $\left.a_{m}\right\}$. We will show (Claim 1 in $\S 3$ ) that (A1) implies that $N(A)$ is nonempty and, further, that it is a test set for the integer programs (1.1).

Condition (A1) implies that there exists a nonzero vector in $D(A)$ with $n+1$ or fewer positive components. Our next condition, a weak form of nondegeneracy of $A$, says

every nonzero $y \in D(A)$ has at least $n+1$ positive components,

which is the same as saying that 0 is not in the convex hull of any $n$ rows of $A$. We will show in Theorem 3 that, under (A1) and (A2), $N(A)$ is finite in a quantitative form.

Finiteness of $N(A)$ was proved in White (1983) and in Bárány, et al. (1995) under the stronger condition "all $n$ by $n$ minors of $A$ are nonsingular."

In general, the set of neighbors need not form a minimal test set for the integer programs (1.1); a proper subset of $N(A)$ may also be a test set. The reason for this ambiguity is that we may have two bodies $\langle 0, h\rangle$ and $\left\langle 0, h^{\prime}\right\rangle$, with distinct lattice points $h$ and $h^{\prime}$, which are identical, free of interior lattice points, but with $h^{\prime}$ on the boundary of the first body and $h$ on the boundary of the second. In this case, removal of either one of these points $h$ or $h^{\prime}$ results in a smaller test set. As we shall see, this is more a problem of exposition than substance, aside from a lower dimensional set of matrices.

The matrix $A$ is called generic if it satisfies conditions (A1) and (A2) and

$$
a_{i} h \neq 0 \text { for every } i \text { and every } h \in N(A) \text {. }
$$

For a generic matrix $A, N(A)$ is the unique minimal test set for (1.1). Notice that generic matrices form a dense set in the collection of matrices satisfying (A1) and (A2): any such matrix with algebraically independent entries is automatically generic.

Now let $A$ be a generic matrix and $C(A)$ the collection of matrices $A^{\prime}$ satisfying, for every $i$ and every $h \in N(A)$

$$
\operatorname{sign} a_{i}^{\prime} h=\operatorname{sign} a_{i} h
$$

As we shall see the closure of $C(A)$ is a polyhedral cone. This follows from 
THEOREM 1. Let $A$ be a generic matrix and $A^{\prime} \in C(A)$. Then $A^{\prime}$ is also generic and has precisely the same set of neighbors as $A$.

This, of course, shows that $C(A)=C\left(A^{\prime}\right)$. Theorem 1 says, in other words, that elements of $C(A)$ are characterized by conditions (cf. (2.1))

$$
a_{i}^{\prime} \in \operatorname{int} C_{i}^{*}, \quad i=1, \ldots, m
$$

where $C_{i}^{*}$ is the polar of the cone $C_{i}$ defined in (1.3). Thus $C(A)$ has a product structure: any choice $a_{i}^{\prime} \in$ int $C_{i}^{*}\left(i=1, \ldots, m\right.$, the $a_{i}^{\prime}$ are chosen independently!) gives rise to a generic matrix $A^{\prime}=\left[a_{1}^{\prime}, \ldots, a_{m}^{\prime}\right]^{T} \in C(A)$.

Write now $G_{i}$ for the set of generators of the cone $C_{i}$. Each $G_{i}$ is finite and

$$
C_{i}^{*}=\left\{x: g x \leq 0, g \in G_{i}\right\}
$$

is a (minimal) polyhedral description of $C_{i}^{*}$ and of $C(A)$. The simpler the structure of the $G_{i}$, the simpler this polyhedral description becomes.

We have investigated the structure of $N(A)$ on several examples, mainly in dimension 3,4 , and 5 . The computational experiments provided beautiful pictures and insightful examples, and showed structural properties of the neighbors. The experiments led to the conjecture that the cones $C_{i}$ have "few" generators. We prove this in the first nontrivial case.

THEOREM 2. If $A$ is a generic 4 by 3 matrix, then the cone $C_{i}$ has either three or four generators and they form the Hilbert basis of $C_{i}$.

We recall from Schrijver (1986) that $H \subset \mathbb{Z}^{n}$ is a Hilbert basis of the pointed polyhedral cone $C \subset R^{n}$ if every $z \in C \cap \mathbb{Z}^{n}$ is a nonnegative integral combination of some elements of $H$ and $H$ is minimal (to containment) with respect to this property. It is known that the Hilbert basis exists and is unique.

Before proceeding to the proofs some remarks are in place here.

REMARK 1. Most frequently, test sets are considered when the corresponding matrix $A$ is integral (Lovász 1989, Sturmfels and Thomas 1994, and others). These matrices often lie on the boundary of the decomposition ( given by Theorem 1 ) of the set of matrices satisfying (A1). For matrices on the boundary of a cell $C$ the set of neighbors need not be a minimal test set.

REMARK 2. In the 4 by 3 case the number of generators of $C_{i},\left|G_{i}\right|$, is bounded independently of $A$ (according to Theorem 2). There is a series of examples (with $n=3$ and $m=5$ ) showing $\left|G_{i}\right|$ is not bounded by a function of $n$ and $m$ alone. However, as A. Barvinok (1995) pointed out, a deep result of R. Kannan (1990) shows that $\left|G_{i}\right|$ is polynomial in the size of $A$. We mention further that, in the 4 by 3 case, in every computational example the generators formed a parallelogram whenever there were four of them.

REMARK 3. The cones $C_{i}$ play a role in another question as well. Sturmfels and Thomas (1994) considered integer programs of the form $\min \left\{c x: A x \leq b, x \in \mathbb{Z}^{n}\right\}$ with $c$ and $b$ varying while $A$ is a fixed national (or integral) matrix. They show that there is a fan, i.e., a subdivision of $R^{n}$ into cones $K_{1}, \ldots, K_{k}$ with nice intersection properties, such that for every $b \in R^{m}$ and every $c_{i}, c_{i}^{\prime} \in$ int $K_{i}$, the integer programs

$$
\begin{gathered}
\min \left\{c_{i} x: A x \leq b, x \in \mathbb{Z}^{n}\right\} \quad \text { and } \\
\min \left\{c_{i}^{\prime} x: A x \leq b, x \in \mathbb{Z}^{n}\right\}
\end{gathered}
$$


have the same solution. It can be shown (using the results of this paper) that for any particular $c_{i} \in$ int $K_{i}, K_{i}$ is the polar of $\operatorname{pos}\left\{h \in N\left(A_{i}\right): c_{i} h<0\right\}$ where $A_{i}=\left[c_{i}, a_{1}\right.$, $\left.\ldots, a_{m}\right]^{T}$.

REMARK 4. There is yet another case where the cones $C_{i}$ come up. Given a generic $m \times n$ matrix $A$ and $b \in R^{m}$ the set $K_{b}$ of the form (1.3) is a maximal lattice free convex body if $\mathbb{Z}^{n} \cap$ int $K_{b}=\phi$ but $\mathbb{Z}^{n} \cap$ int $K=\phi$ for every convex body $K$ properly containing $K_{b}$. Every facet of $K_{b}$ contains exactly one lattice point in its relative interior. Associating this set of lattice points with the maximal lattice free convex body $K_{b}$ gives rise to a simplicial complex $K(A)$ depending only on $A$ (see Bárány et al. (1994) and Bárány et al. (1995) for the precise definition). The proof of Theorem 1 shows that for $A^{\prime} \in C(A)$, the simplicial complexes $K(A)$ and $K\left(A^{\prime}\right)$ coincide.

3. $N(A)$ is nonempty and finite. We show first that, under condition $(\mathrm{A} 1), N(A)$ is nonempty in the following stronger form.

Claim 1. If $A$ satisfies (A1), then every set $K_{b}$ with $0 \in K_{b}$ and $\left|\mathbb{Z}^{n} \cap K_{b}\right| \geq 2$ contains a neighbor of $A$.

Proof. Suppose $0, z \in \mathbb{Z}^{n} \cap K_{b}, z \neq 0$. We construct a (finite) sequence $z=z_{0}, z_{1}$, $\ldots, z_{l}$ so that $z_{i} \in \operatorname{int}\left\langle 0, z_{i-1}\right\rangle,\left\langle 0, z_{i}\right\rangle \subset\left\langle 0, z_{i-1}\right\rangle(i=1, \ldots, l)$ and $z_{l} \in N(A)$.

Assume $z_{i}$ has been constructed. If $\mathbb{Z}^{n} \cap$ int $\left\langle 0, z_{i}\right\rangle=\phi$, set $l=i$ and stop. Otherwise pick any $z_{i+1} \in \mathbb{Z}^{n} \cap$ int $\left\langle 0, z_{i}\right\rangle$ and continue. The algorithm stops since, in view of (A1), $K_{b}$ is bounded and $z_{0}, z_{1}, \ldots, z_{l}$ all belong to $\left\langle 0, z_{0}\right\rangle \subset K_{b}$.

The claim implies that $N(A) \neq \phi$ and, further, that $N(A)$ is a test set for the integer programs (1.1). Now we turn to the proof of finiteness of $N(A)$.

As $N(A)$ does not change if $a_{i}$ is multiplied by a positive number we may and do assume that $\left\|a_{i}\right\|=1$ for all $i$. Define

$$
d=\min \{|\operatorname{det} B|: B \text { is a nonsingular } n \times n \text { minor of } A\} .
$$

THEOREM 3. If A satisfies (A1) and (A2), then for every $h \in N(A)$

$$
\|h\| \leq \frac{n^{2}}{d}
$$

Proof. We are indebted to Martin Henk for a remark simplifying our original proof of this theorem.

Fix $h \in N(A),\langle 0, h\rangle$ is bounded (by (A1)) and int $\langle 0, h\rangle \neq \phi$ because of (A2). Consider the ball $B$ inscribed in $\langle 0, h\rangle$ that has the largest radius $\rho$, let its center be $c$.

$\mathbb{Z}^{n} \cap B=\phi$ implies, via a simple induction, that $\rho \leq \frac{1}{2} \sqrt{n}$.

Write $I$ for the set of indices $i \in\{1, \ldots, m\}$ for which the hyperplane $\left\{x: a_{i} x=b_{i}\right\}$ is tangent to $B$. (Here $b_{i}=\max \left\{0, a_{i} h\right\}$.) For $i \in I$ the equation of this hyperplane can be written as

$$
a_{i}(x-c)=\rho .
$$

The corresponding inequalities represent the "active" constraints on the largest inscribed ball. The simple necessary condition for the maximality of $\rho$ is $0 \in \operatorname{conv}\left\{a_{i}: i\right.$ $\in I\}$. Then condition (A2) implies $0 \in$ int $\operatorname{conv}\left\{a_{i}: i \in I\right\}$ which shows, in turn, that the polyhedron 


$$
P=\left\{x: a_{i} x \leq b_{i}, i \in I\right\}
$$

is bounded (and, further, that $B$ is unique but we won't need this). Clearly $\langle 0, h\rangle \subset P$.

A vertex $v$, of $P$, is the solution of $n$ equations of the form (3.3). Write $M$ for the matrix whose rows are the $a_{i}$ of these $n$ equations. Further, let $M^{j}$ be the matrix obtained from $M$ by replacing its $j$ th column by the all-one column. We get for the $j$ th component of $v$ $-c$

$$
(v-c)_{j}=\rho \frac{\operatorname{det} M^{j}}{\operatorname{det} M}
$$

The denominator here is nonzero since otherwise the corresponding equations do not determine a vertex. Expanding the numerator along the all-one column and using $\left\|a_{i}\right\|$ $\leq 1$ we get $\left|(v-c)_{i}\right| \leq \rho n / d$. By $(3.1)$

$$
\|v-c\| \leq \rho n \sqrt{n} / d \leq n^{2} / 2 d .
$$

But $\operatorname{diam}\langle 0, h\rangle \leq \operatorname{diam} P \leq n^{2} / d$ because the diameter of $P$ occurs between two of its vertices.

4. Proof of Theorem 1. We start the argument by taking $A^{\prime}$ to be identical with $A$ in rows $2, \ldots, m$ and differing only in row 1 . By assumption sign $a_{1}^{\prime} h=\operatorname{sign} a_{1} h$ for every $h \in N(A)$.

\section{Claim 2. $\quad N\left(A^{\prime}\right) \subset N(A)$.}

Proof. Let $h^{\prime} \in N\left(A^{\prime}\right)$. There is no loss in generality in assuming that $a_{1} h^{\prime} \leq 0$ since if this were not true we could select the neighbor $-h^{\prime} \in N\left(A^{\prime}\right)$.

Assume $h^{\prime}$ is not a neighbor of $A$. Then by Claim 1 of the previous section there is an $h \in N(A)$ with $h \in \operatorname{int}\left\langle 0, h^{\prime}\right\rangle_{A}$, so that

$$
\begin{gathered}
a_{1} h<\max \left\{0, a_{1} h^{\prime}\right\}=0, \\
a_{i} h<\max \left\{0, a_{i} h^{\prime}\right\}=\max \left\{0, a_{i}^{\prime} h^{\prime}\right\}, \quad i=2, \ldots, m .
\end{gathered}
$$

We show now that $h \in \operatorname{int}\left\langle 0, h^{\prime}\right\rangle_{A^{\prime}}$ contradicting the assumption that $h^{\prime} \in N\left(A^{\prime}\right)$.

We certainly have $a_{i}^{\prime} h=a_{i} h<\max \left\{0, a_{i}^{\prime} h^{\prime}\right\}$ for $i=2, \ldots, m$. In order to demonstrate $a_{1}^{\prime} h<\max \left\{0, a_{1}^{\prime} h^{\prime}\right\}$ it suffices to show that $a_{1}^{\prime} h<0$. But since $h \in N(A)$ we have sign $a_{1}^{\prime} h=\operatorname{sign} a_{1} h<0$.

Write now $A(t)=t A+(1-t) A^{\prime}$ and $a_{1}(t)=t a_{1}+(1-t) a_{1}^{\prime}$. We use a homotopy argument for

LEMMA 1. $A(t)$ is generic for every $t \in[0,1]$.

We show first how this implies Theorem 3. $A^{\prime}=A(1)$ is generic by Lemma 1. Further, sign $a_{i}^{\prime} h=\operatorname{sign} a_{i} h$ for every $i$ and every $h \in N\left(A^{\prime}\right)$ since $N\left(A^{\prime}\right) \subset N(A)$ by Claim 2 . Claim 2 applies again with the roles of $A$ and $A^{\prime}$ interchanged showing $N(A)=N\left(A^{\prime}\right)$.

To finish the proof of Theorem 1 we repeat the same argument for every row in $A$.

Proof of Lemma 1. Set

$$
t^{*}=\min \{t \geq 0: A(t) \text { is not generic }\} \text {. }
$$


where the existence of the minimum and $t^{*}>0$ are easily justified. Assume, by way of contradiction, that $t^{*} \leq 1$. Clearly sign $a_{1}(t) h=\operatorname{sign} a_{1} h$ for every $h \in N(A)$ and every $t \in[0,1]$. Thus $A(t)$ satisfies condition (A3) for every $t \in[0,1]$. Claim 2 implies, further, $N(A(t)) \subset N(A)$ for every $t \in\left[0, t^{*}\right)$.

We can reformulate conditions (A1) and (A2) for $A(t)$ as

(A1') $0 \in$ int $\operatorname{conv}\left\{a_{1}(t), a_{2}, \ldots, a_{m}\right\}$,

(A2') $0 \notin \operatorname{conv}\{$ any $n$ of them $\}$.

These conditions are true for $t \in\left[0, t^{*}\right)$ but one of them fails at $t^{*}$. If ( $\left.\mathrm{A} 1^{\prime}\right)$ fails, then 0 appears on the boundary of conv $\left\{a_{1}\left(t^{*}\right), a_{2}, \ldots, a_{m}\right\}$. By Caratheodory's theorem, 0 is in the relative interior of the convex hull of some of these vectors, including, of course, $a_{1}\left(t^{*}\right)$. Renaming these vectors suitably we get

$$
0 \in \text { relint } \operatorname{conv}\left\{a_{1}\left(t^{*}\right), a_{2}, \ldots, a_{k}\right\}
$$

where $k \leq n$ and we assume, further, that $a_{2}, \ldots, a_{k}$ are linearly independent.

If (A2') fails at $t^{*}$, then 0 is in the convex hull of some $n$ or fewer of the rows of $A\left(t^{*}\right)$. We conclude again, that (4.1) holds with $k \leq n$ and $a_{2}, \ldots, a_{k}$ linearly independent.

ClaIM 3. There are $n+1-k$ rows of $A(t)$ which we can take to be $a_{k+1}, \ldots, a_{n+1}$ so that for all $t \in\left[0, t^{*}\right)$

$$
0 \in \text { int } \operatorname{conv}\left\{a_{1}(t), a_{2}, \ldots, a_{n+1}\right\} .
$$

Again, we show first how Lemma 1 follows from here. Claim 3 and (A1') imply that the cone

$$
C(t)=\left\{x \in R^{n}: a_{1}(t) x<0, a_{2} x<0, \ldots, a_{n} x<0\right\}
$$

is simplicial and nonempty. Then

$$
\min \left\{a_{n+1} z: z \in C(t) \cap \mathbb{Z}^{n}\right\}
$$

is reached at some $h(t) \in C(t) \cap \mathbb{Z}^{n}$. Since $h(t)$ is a neighbor for the matrix [ $a_{1}(t), a_{2}$, $\left.\ldots, a_{n+1}\right]^{T}$, it is a neighbor for $A(t)$ as well. By Claim $2, h(t) \in N(A)$. As $N(A)$ is finite, there is a sequence $t_{\mu} \rightarrow t^{*}($ as $\mu \rightarrow \infty)$ so that $h\left(t_{\mu}\right)=h \in N(A)$ for all $\mu$. Thus $a_{1}\left(t^{*}\right) h<0, a_{2} h<0, \ldots, a_{k} h<0$ showing that the hyperplane $\{x: h x=0\}$ strictly separates 0 from $\left\{a_{1}\left(t^{*}\right), a_{2}, \ldots, a_{k}\right\}$. This contradicts (4.1) and finishes the proof of Lemma 1.

The proof of Claim 3 is technical but rather straightforward: We are going to find $a_{k+1}$, $\ldots, a_{n+1}$ by projecting along the subspace $L=\operatorname{lin}\left\{a_{2}, \ldots, a_{k}\right\}=\operatorname{lin}\left\{a_{1}\left(t^{*}\right), a_{2}, \ldots\right.$, $\left.a_{k}\right\}$. Let $\bar{x}$ denote the orthogonal projection of $x \in R^{n}$ onto $L^{\perp}$, the orthogonal complement of $L$. Set $Q(t)=\operatorname{conv}\left\{\bar{a}_{1}(t), \bar{a}_{k+1}, \ldots, \bar{a}_{m}\right\}$. (A1) implies

$$
0 \in \text { relint } Q(t) \text { for } t \in\left[0, t^{*}\right) .
$$

The halfline $\left\{-\lambda \bar{a}_{1}(t): \lambda \geq 0\right\}$ intersects the boundary of $Q(t)$ (which is a convex polytope in $\left.L^{\perp}\right)$ at $-\lambda(t) \bar{a}_{1}(t)$. This point belongs to a facet $F(t)$ of $Q(t)$. Since $\bar{a}_{1}(t)$ is not on this facet and since $\bar{a}_{1}(t)$ changes linearly with $t, F(t)$ is constant on an interval $\left[t^{\prime}, t^{*}\right)$. By Caratheodory's theorem there are linearly independent vertices of $F(t)$, which we take to be $\bar{a}_{k+1}, \ldots, \bar{a}_{p}$, such $-\lambda(t) \bar{a}_{1}(t) \in \operatorname{conv}\left\{\bar{a}_{k+1}, \ldots, \bar{a}_{p}\right\}$ implying 


$$
-\bar{a}_{1}(t)=\sum_{i=k+1}^{p} \alpha_{i}(t) \bar{a}_{i}
$$

with $\alpha_{i}(t)$ continuous on $\left[t^{\prime}, t^{*}\right]$, positive on $\left[t^{\prime}, t^{*}\right)$, and 0 at $t^{*}$. The linear independence of $\bar{a}_{k+1}, \ldots, \bar{a}_{p}$ shows $p \leq n+1$.

Lifting (4.3) back to $R^{n}$ we get

$$
-a_{1}(t)=l(t)+\sum_{k+1}^{p} \alpha_{i}(t) a_{i}
$$

where $l(t) \in L$ so that $l(t)=\Sigma_{2}^{k} \alpha_{i}(t) a_{i}$ with uniquely determined and continuous ( since $l(t)$ is continuous ) coefficients $\alpha_{i}(t)$. We then have

$$
0=a_{1}(t)+\sum_{2}^{p} \alpha_{i}(t) a_{i} .
$$

Here $\alpha_{i}(t)>0$ for $i>k$, and $\alpha_{i}(t)>0$ for $i=2, \ldots, k$ on $\left[t^{\prime \prime}, t^{*}\right)$ as well since $\alpha_{i}\left(t^{*}\right)$ $>0$ as follows from (4.1).

(4.4) shows $0 \in$ relint $\operatorname{conv}\left\{a_{1}(t), \ldots, a_{p}\right\}$ when $t \in\left[t^{\prime \prime}, t^{*}\right)$. By (A2') $p=n+1$ and $0 \in$ int $\operatorname{conv}\left\{a_{1}(t), \ldots, a_{p+1}\right\}$. By $\left(\mathrm{A} 2^{\prime}\right)$, again, this holds for all $t \in\left[0, t^{*}\right)$.

5. Few generators. From now on we work with the $4 \times 3$ case. The arguments of the next two sections provide a proof of Theorem 2.

Shallcross (1992) has given a complete characterization of the neighbors in this case. Although we do not use this characterization explicitly, it provides considerable insight. Claims 1 and 2 below can be found in Shallcross (1992) as well.

With a slight change of notation let $a_{0}, a_{1}, a_{2}, a_{3}$ be the rows of $A$. We assume again that $A$ is generic. Define $H_{i}^{0}, H_{i}^{+}, H_{i}^{-}$as the set of $x \in R^{3}$ with $a_{i} x=0,>0,<0$ respectively.

We are interested in the neighbors $N=\left\{h \in N(A): a_{0} h<0\right\}$. They lie in cones of the type $H_{0}^{-} \cap H_{1}^{+} \cap H_{2}^{+} \cap H_{3}^{-}$which we denote by $C_{12}$ : the index shows which of the $H_{i}$ go with + superscript. By condition (A1) $H_{0}^{-} \cap H_{1}^{-} \cap H_{2}^{-} \cap H_{3}^{-}=\varnothing$. So the cones in question are $C_{1}, C_{2}, C_{3}, C_{12}, C_{23}, C_{31}$, and $C_{123}$.

Observe that the cones $C_{1}, C_{2}, C_{3}$, and $C_{123}$ contain exactly one neighbor, to be denoted by $s_{1}, s_{2}, s_{3}$, and $s_{0}$, respectively. To see this note that, for instance $s_{2}$ is the unique solution to the integer program

$$
\min \left\{a_{2} x: a_{i} x<0, i=0,1,3, x \in \mathbb{Z}^{3}\right\} .
$$

Since multiplying $a_{i}$ by a positive number does not change the neighbors we may assume that $a_{0} s_{0}=-1$ and $a_{i} s_{i}=1(i=1,2,3)$. Set

$$
Q=\left\{x \in R^{3}:\left|a_{i} x\right| \leq 1, i=0,1,2,3\right\} .
$$

Claim 1. $N(A) \subset Q$.

Proof. Assume $h \in N(A)$ but $h \notin Q, a_{0} h>1$, say. As $h$ is a neighbor, there is no integer other than 0 and $h$ satisfying $a_{i} x \leq \max \left\{a_{i} h, 0\right\}$ for all $i$. But $-s_{0}$ satisfies all these inequalities since $a_{0}\left(-s_{0}\right)=1<a_{0} h$ and $a_{i}\left(-s_{0}\right)<0$ when $i=1,2,3$.

Recall now the definition of $C=\operatorname{pos} N$ and write $D=C \cup(-C)$. We know from Theorem 1 that $a_{0}$ can be moved without changing $N(A)$ as long as $H_{0}$ does not meet $C$. 
CLAIM 2. $Q \backslash D$ contains no lattice point.

Proof. Assume to the contrary that there is a point $z \in \mathbb{Z}^{3} \cap Q \backslash D$. Move $a_{0}$ along $a_{0}(t)=a_{0}+t a$ until $H_{0}(t)$ passes through the first such lattice point $z$. This happens at $t=t_{0}$, say. Since $z$ is not a neighbor, it is in one of the cones $C_{12}, C_{23}$, or $C_{31}$, say $C_{12}$. But as $H_{0}(t)$ passes through $z$, it will be in the cone $H_{0}^{+}(t) \cap H_{1}^{+} \cap H_{2}^{+} \cap H_{3}^{-}$, which contains the unique neighbor $-s_{3}$. So $z=-s_{3}$, a contradiction.

ClaIM 3. If $u$ and $v$ are generators of $C$, then $u-v \notin Q$.

Proof. If $u-v \in Q$ then, by Claim $2, u-v$ is either in $C$ or in $-C$. Assuming $u$ $-v \in C, u \in v+C$, so $u=v+c$ for some $c \in C$. But then $u$ is not a generator of $C$.

Now if $u, v \in C \cap Q$ belong to the same cone $C_{12}, C_{23}$, or $C_{31}$, then automatically $u$ $-v \in Q$. This shows that $C$ can have at most seven generators, one in each of the cones $C_{i}, C_{i j}, C_{123}$. The trivial observation $s_{0} \in \operatorname{pos}\left\{s_{1}, s_{2}, s_{3}\right\}$ implies that $C$ has at most six generators. The next claim takes this number down to four.

ClaIM 4. If $s_{1}$ and $s_{2}$ are generators of $C$, then $C$ has no generator in $C_{12}$.

Proof. Assume $h \in N \cap C_{12}$ is such a generator. We will show that $a_{i}\left(s_{1}+s_{2}\right)$ $\leq \max \left\{a_{i} h, 0\right\}$ for $i=0,1,2,3$ contradicting $h \in N$. First, for $i=0$ or $i=3$

$$
a_{i}\left(s_{1}+s_{2}\right)<0=\max \left\{a_{i} h, 0\right\} .
$$

By Claim 3, $s_{1}-h \notin Q$. Now $\left|a_{3}\left(s_{1}-h\right)\right|<1$ clearly, and $a_{1}\left(s_{1}-h\right)=1-a_{1} h \in(0$, 1) since $h \in Q$. Further, $a_{0}$ can be moved without changing $N$ so that $H_{0}$ almost contains $s_{1}$ and $h$. This follows from Theorem 2 and the fact that $s_{1}$ and $h$ are consecutive generators of $C$. Then $a_{0}\left(s_{1}-h\right)$ is between -1 and 1 . Consequently, $a_{2}\left(s_{1}-h\right)<-1$. So we get $a_{2}\left(s_{1}+s_{2}-h\right)=a_{2} s_{2}+a_{2}\left(s_{1}-h\right)<0$, i.e.,

$$
a_{2}\left(s_{1}+s_{2}\right)<a_{2} h=\max \left\{a_{2} h, 0\right\} .
$$

One proves $a_{1}\left(s_{1}+s_{2}\right)<a_{1} h=\max \left\{a_{1} h, 0\right\}$ the same way.
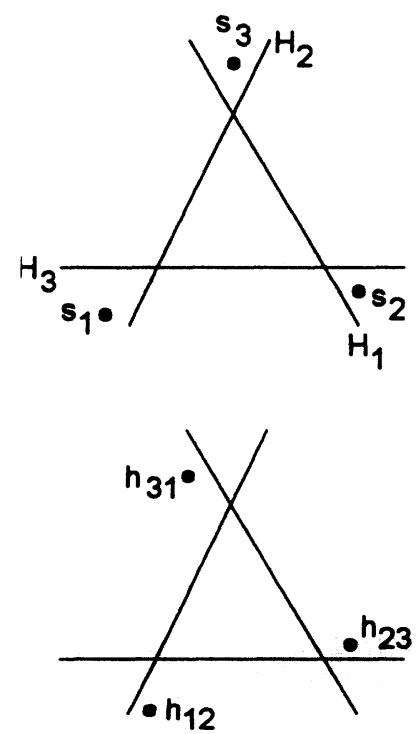
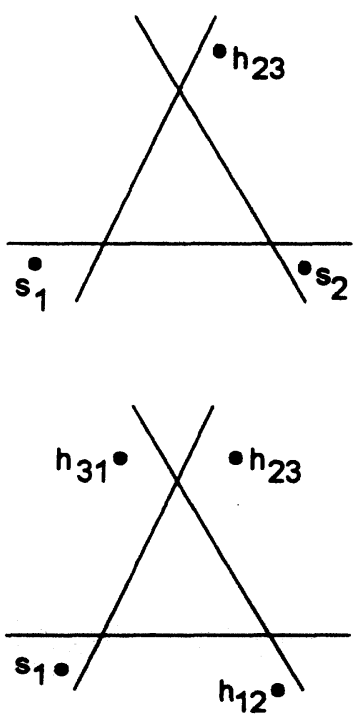

FIGURE 1
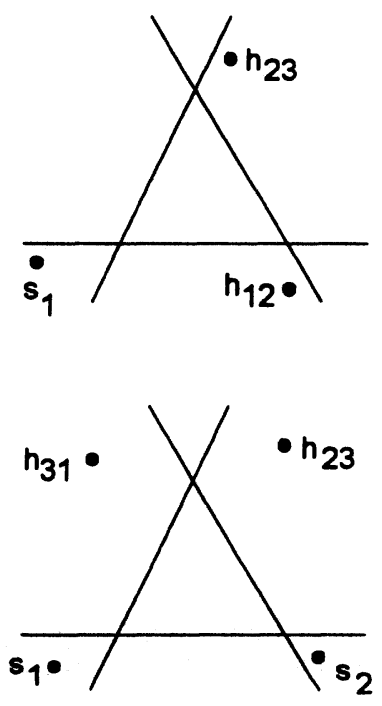
The figure presents the remaining six cases in the plane $a_{0} x=-1$; the three lines are the traces of the planes $H_{1}, H_{2}, H_{3}$.

\section{The structure of the generators.}

Claim 1. If $u, v$ are generators of $C$, then $u, v$ form a basis of the lattice $\mathbb{Z}^{3}$ $\cap \operatorname{lin}\{u, v\}$.

Proof. By Claim 2 of the previous section there is no integer in the triangle $[0, u$, $-v]$ other than its vertices. Consequently $[0, u,-v, u-v]$ is a lattice parallelogram.

Here and in what follows we write $[a, b, c, d]$ for the convex hull of $a, b, c, d \in R^{3}$. We say that $[a, b, c, d]$ is special if it contains no lattice point other than $a, b, c, d$. The notation and terminology are extended to triangles and segments as well.

ClAIM 2. If $u, v, w$ are consecutive generators of $C$, then $[0, u, v,-w]$ and $[0,-u$, $v, w]$ are special simplices.

Proof. This is true because of the previous claim and because the simplices in question are contained in $Q \backslash D$.

LEMMA 2. If $0, a, b, c \in \mathbb{Z}^{3}$ are not coplanar and the simplices $[0, a, a+b, a+c]$ and $[0, c, a+c, b+c]$ are special, then so are $[a+b+c, b+c, c, b],[a+b+c$, $a+b, b, a]$. Moreover, all lattice points in $T=\{\alpha a+\beta b+\gamma c: 0<\alpha, \beta, \gamma<1\}$ are of the form $\alpha(a+c)+\beta b$ for some $\alpha, \beta \in(0,1)$.

PROOF. The first statement follows simply by reflection through $\frac{1}{2}(a+b+c)$. The second needs more meditation.

Obviously, $a$ and $c$ generate the lattice $\mathbb{Z}^{3} \cap \operatorname{lin}\{a, c\}$. Then we can pick $z \in T \cap \mathbb{Z}^{3}$ so that $a, c, z$ form a basis of $\mathbb{Z}^{3}$. Thus

$$
b=\lambda_{1} a+\lambda_{2} c+\lambda_{3} z
$$

with $\lambda_{i}$ an integer. In fact $\lambda_{3}>1$ since $\lambda_{3}=1$ would mean that $a, b, c$ form a basis of $\mathbb{Z}^{3}$ and then $\mathbb{Z}^{3} \cap$ int $T=\varnothing$. Since $z \in T$ and $\lambda_{3} z=b-\lambda_{1} a-\lambda_{2} c, \lambda_{1}<0$ and $\lambda_{2}<0$.

Clearly $z \in \operatorname{pos}\{a, b, c\}$ and the conditions concerning special simplices imply $z$ $\in \operatorname{pos}\{a+b, b+c, c+a, b\}$. This cone is the union of $\operatorname{pos}\{a+b, b+c, b\}$ and $\operatorname{pos}\{a+b, b+c, c+a\}$.

If $z \in \operatorname{pos}\{a+b, b+c, b\}$, then we have, with $\mu_{i}>0$,

$$
\begin{aligned}
\lambda_{3} z & =\mu_{1}(a+b)+\mu_{2}(b+c)+\mu_{3} b \\
& =\left(\mu_{1}+\mu_{2}+\mu_{3}\right) b+\mu_{1} a+\mu_{2} c=b-\lambda_{1} a-\lambda_{2} c .
\end{aligned}
$$

So $\mu_{i}=-\lambda_{i}(i=1,2)$ are positive integers and $\mu_{1}+\mu_{2}+\mu_{3}=1$ with $\mu_{3}>0$ which is impossible.

Therefore we have $z \in \operatorname{pos}\{a+b, b+c, c+a\}$, and again with positive $\mu_{i}$

$$
\lambda_{3} z=b-\lambda_{1} a-\lambda_{2} c=\mu_{1}(a+b)+\mu_{2}(b+c)+\mu_{3}(c+a) .
$$

The solution is $\mu_{1}=\frac{1}{2}\left(1-\lambda_{1}+\lambda_{2}\right)$ and $\mu_{2}=\frac{1}{2}\left(1+\lambda_{1}-\lambda_{2}\right)$ which is possible with positive $\mu_{i}$ and integer $\lambda_{i}$ if and only if $\lambda_{1}=\lambda_{2}$. This proves the lemma.

We return now to the proof of Theorem 3. Write $G$ for the set of generators of $C$. If $a, b, c$ are consecutive generators of $C$, then $[0, a, b,-c]$ and $[0,-a, b, c]$ are special 
(by Claim 2). Then so are $[c, a+c, b+c, 0]$ and $[a, 0, a+b, a+c]$ (by translation) and Lemma 3 applies.

Consider first the case $|G|=3$. Then $a, b, c$ are consecutive generators in every order and, by Lemma 3, each integer in $T$ is of the form $\alpha(a+b+c)$. So we have

Corollary. If $C$ has three generators $a, b, c$ with $|\operatorname{det}(a, b, c)|=\lambda>1$, then $T$ $\cap \mathbb{Z}^{3}=\{k(a+b+c) / \lambda$ for $k=1, \ldots, \lambda-1\}$.

We are to check the first four cases of the figure separately.

CASE 1. $G=\left\{s_{1}, s_{2}, s_{3}\right\}$. If $s_{1}+s_{2} \notin Q$, then $a_{3}\left(s_{1}+s_{2}\right)<-1$ must hold since $a_{0} s_{1}$ and $a_{0} s_{2}$ can be taken almost equal to zero. So if $s_{1}+s_{2} \notin Q$ then $a_{3}\left(s_{1}+s_{2}+s_{3}\right)$ $<0$. Similarly, $s_{2}+s_{3} \notin Q$ and $s_{3}+s_{1} \notin Q$, respectively, imply $a_{1}\left(s_{1}+s_{2}+s_{3}\right)<0$ and $a_{2}\left(s_{1}+s_{2}+s_{3}\right)<0$. Since $a_{0}\left(s_{1}+s_{2}+s_{3}\right)<0$ automatically, and $a_{i}\left(s_{1}+s_{2}+s_{3}\right)$ $<0$ for $i=0,1,2,3$ contradicts (A1) we must have either $s_{1}+s_{2} \in Q$ or $s_{2}+s_{3} \in Q$ or $s_{3}+s_{1} \in Q$. Assume, say, $s_{1}+s_{2} \in Q$. Then the interior of the segment $\left[-s_{3}, s_{1}+s_{2}\right]$ lies in $Q \backslash D$ so the segment is special. But then $s_{3}+\left[-s_{3}, s_{1}+s_{2}\right]=\left[0, s_{1}+s_{2}+s_{3}\right]$, is also special and the corollary implies $\operatorname{det}\left(s_{1}, s_{2}, s_{3}\right)= \pm 1$.

CASE 2. $G=\left\{s_{1}, s_{2}, h_{23}\right\}$. Then $s_{1}+h_{23} \in Q$ and the segment $\left[-s_{2}, s_{1}+h_{23}\right]$ $\in Q \backslash D$. The same argument as above shows that $\operatorname{det}\left(s_{1}, s_{2}, h_{23}\right)= \pm 1$.

CASE 3. $G=\left\{s_{1}, h_{12}, h_{23}\right\}$. Again $s_{1}+h_{23} \in Q$ and the segment $\left[-h_{12}, s_{1}+h_{23}\right]$ $\in Q \backslash D$, and we repeat the above argument.

CASE 4. $G=\left\{h_{12}, h_{23}, h_{31}\right\}$. We are done again if $h_{12}+h_{23} \in Q$. If none of $h_{12}$ $+h_{23}, h_{23}+h_{31}$, and $h_{31}+h_{12}$ is in $Q$, then $h_{12}+h_{23}+h_{31} \in C_{123}$ as one can easily check. Let $z=\left(h_{12}+h_{23}+h_{31}\right) / \lambda$ be the first integral point on the diagonal of $T$, where, of course, $\lambda \in \mathbb{Z}$ and assume $\lambda \geq 2$. Then

$$
1=\left|a_{0} s_{0}\right| \leq\left|a_{0} z\right|=\frac{1}{\lambda}\left|a_{0}\left(h_{12}+h_{23}+h_{31}\right)\right|
$$

But since $a_{0}$ can be moved so that $a_{0} h_{12}$ and $a_{0} h_{31}$ are almost zero, we get

$$
1 \leq \frac{1}{\lambda}\left|a_{0} h_{23}\right| \leq \frac{1}{\lambda}\left|a_{0} s_{0}\right|=\frac{1}{\lambda} .
$$

Assume now that $C$ has four generators $a, b, c, d$ in this order. The relative interiors of the cones pos $\{a, c\}$ and $\operatorname{pos}\{b, d\}$ intersect so we have

$$
\alpha a+\gamma c=\beta b+\delta d
$$

with $\alpha, \beta, \gamma, \delta>0$ and we may assume, by multiplying if necessary, that the smallest coefficient is equal to one.

We show first that either $\alpha=\gamma$ or $\beta=\delta$. By symmetry we may assume that $\beta$ is maximal among $\alpha, \beta, \gamma, \delta$. Either $\beta=\delta$ and we are done or $\beta>\delta$ and we get

$$
b+d=\left(1-\frac{\delta}{\beta}\right) b+\frac{\alpha}{\beta} a+\frac{\gamma}{\beta} c
$$

and this point is in $T$ since all the coefficients on the right hand side are between 0 and 1. So Lemma 3 shows that $\alpha=\gamma$.

Claim 2 applies to $a, b, c$ and $b, c, d$ and $c, d, a$ and $d, a, b$ showing that $[0, a, b$, $-c],[0, b,-c,-d]$, and $[0,-c,-d, a][0,-d, a, b]$ are special. This implies that 
their union, $[0, a, b,-c,-d]$ is also special which imposes a restriction. Namely, by a theorem in Scarf (1986), these five points must lie on two consecutive lattice hyperplanes, $H_{1}, H_{2}$, say. If four of them lie in one of the hyperplanes, then they have to be $a, b,-c$, $-d$ as otherwise three of the generators would lie in a hyperplane through the origin. But then $a, b, c, d$ are the vertices of a (special) parallelogram and they clearly form the Hilbert basis of $C$. We may assume now that the split is two-three and $0 \in H_{1}$. By symmetry it is enough to consider three cases.

CASE 1. $0, a, b \in H_{1}$ and $-c,-d \in H_{2}$. Then $a, b$ generate $\mathbb{Z}^{3} \cap \operatorname{lin}\{a, b\}$. Consequently $a, b, c$ and $a, b, d$ form a basis of $\mathbb{Z}^{3}$. Further, $\operatorname{lin}\{a, b\}$ contains $c-d$. So $c-d=x a+y b$ with integer $x$ and $y$. This gives $c+(-x) a=y b+d$ but this is the same (apart from a multiplier) as the linear dependence (6.1) where, as we just proved, either $\alpha=\gamma$ or $\beta=\delta$. So either $x=-1$ and $y>0$ or $y=1$ and $x<0$.

In the first case $a+c=y b+d$, and therefore $\operatorname{det}(a, b, d)=\operatorname{det}(b, c, d)$. So both $a, b, d$ and $b, c, d$ form a basis of $\mathbb{Z}^{3}$ and then they are the Hilbert bases of the cones $\operatorname{pos}\{a, b, d\}$ and $\operatorname{pos}\{b, c, d\}$, respectively, whose union is $C$. In the second case $c$ $+(-x) a=b+d$ and the same argument works.

CASE 2. $0, a,-c \in H_{1}$ and $b,-d \in H_{2}$. Then $a,-c$ generate $\mathbb{Z}^{3} \cap \operatorname{lin}\{a,-c\}$. Consequently $a, b, c$ and $a, c, d$ form a basis of $\mathbb{Z}^{3}$. Then they are the Hilbert bases of the cones pos $\{a, b, c\}$ and $\operatorname{pos}\{a, c, d\}$, respectively, whose union is $C$.

CASE 3. $0, a \in H_{1}$ and $b,-c,-d \in H_{2}$. Then $b,-c,-d$, and consequently $b, c$, $d$, form a basis of $\mathbb{Z}^{3}$. Moreover, $b+c, b+d$ form a basis of $\mathbb{Z}^{3} \cap H_{1}$ so $a=x(b+c)$ $+y(b+d)$ with integral $x$ and $y$. Then $a+(-x) c=(x+y) b+y d$ which is the same (apart from a multiplyer) as (6.1). But $\alpha=\gamma$ or $\beta=\delta$ holds as well. That is possible only if $x=-1$. Then $\operatorname{det}(a, b, d)=\operatorname{det}(b, c, d)$ and, again, $a, b, d$ and $b, c, d$ form a basis of the lattice, and they are the Hilbert bases of $\operatorname{pos}\{a, b, d\}$ and $\operatorname{pos}\{b, c, d\}$, respectively, whose union is $C$.

Acknowledgments. The research of the first author is partially supported by Hungarian National Science Foundation Grant No. 4296 and 016937 and Research Grant of the Academy AKP/96-61/13. The research of both authors is supported by the program in Discrete Mathematics of the Cowles Foundation at Yale University.

\section{References}

Bárány, I., R. Howe, H. E. Scarf. 1994. The complex of maximal lattice free simplices. Math. Programming 66 273-281.

- H. E. Scarf, D. Shallcross. 1998. The topological structure of maximal lattice free convex bodies: the general case. Math. Programming 80 1-15.

Barvinok, A. 1995. Personal communication.

Graver, J. E. 1975. On the foundations of linear and integral programming, I. Math. Programming 8 207-266.

Kannan, R. 1990. Test sets for integer programs with $\forall, \exists$ sentences. DI-MACS series, vol. 1. AMS, 39-47.

Lovász, L. 1989. Geometry of numbers and integer programming. M. Iri and K. Tanabe, eds. Mathematical Programming: Recent Developments and Applications. Kluwer Acad., Norwell, MA. 177-210.

$\rightarrow$ Scarf, H. E. 1986. Neighborhood systems for production sets with indivisibilities. Econometrica 54 507-532.

Schrijver, A. 1986. Theory of Linear and Integer Programming. John Wiley \& Sons, New York.

$\rightarrow$ Shallcross, D. 1992. Neighbors of the origin for four by three matrices. Math. Oper. Res. 17 608-614.

Sturmfels, B., R. Thomas. 1994. Variation of cost function in integer programming, manuscript, Cornell University, Ithaca, NY.

White, P. 1983. Discrete activity analysis. Ph.D. Thesis, Yale University, Department of Economics, New Haven, CT.

Imre Bárány: Mathematical Institute of the Hungarian Academy of Sciences, P. O. Box 127, Budapest, 1364 Hungary

Herbert E. Scarf: Cowles Foundation, Yale University, New Haven, Connecticut 06520 